\title{
Effect of Flip Angle on the Correlation Between Signal Intensity and Different Concentrations of Iron Oxide Nanoparticles Using T1-Weighted Turbo-FLASH Inversion Recovery Sequence
}

\author{
Nahideh Gharehaghaji ${ }^{1} ;$ Mahmood Nazarpoor ${ }^{1, *} ;$ Hodaiseh Saharkhiz $^{2}$ \\ ${ }^{1}$ Department of Radiology, Faculty of Paramedicine, Tabriz University of Medical Sciences, Tabriz, Iran \\ ${ }^{2}$ Department of Medical Physics, Faculty of Medicine, Tabriz University of Medical Sciences, Tabriz, Iran \\ ${ }^{*}$ Corresponding author: Mahmood Nazarpoor, Department of Radiology, Faculty of Paramedicine, Tabriz University of Medical Sciences, Tabriz, Iran. Tel: +98-4136681735, \\ Fax: +98-4133368733, E-mail: mnazarpoor@yahoo.co.uk, nazarpoorm@tbzmed.ac.ir
}

Received: August 19, 2014; Revised: October 27, 2014; Accepted: November 4, 2014

\begin{abstract}
Background: Ultrasmall superparamagnetic iron oxide nanoparticles have been used as a blood pool contrast agent for magnetic resonance angiography and perfusion studies. Linear relationship between signal intensity (SI) and nanoparticle concentration is essential for perfusion measurement.

Objectives: The aim of this study was to investigate the effect of different flip angles on maximum SI and the linear relationship between SI and different concentrations of iron oxide nanoparticles using T1-weighted Turbo-FLASH (fast low angle shot) inversion recovery sequence to find the optimum flip angle for perfusion measurement.

Materials and Methods: This in vitro study was performed using carboxydextran coated iron oxide nanoparticles with $20 \mathrm{~nm}$ hydrodynamic size. Different concentrations of nanoparticles between 0 and $500 \mu \mathrm{mol} \mathrm{Fe/L} \mathrm{were} \mathrm{prepared.} \mathrm{MR} \mathrm{imaging} \mathrm{was} \mathrm{performed}$ using T1-weighted Turbo-FLASH inversion recovery sequence. Applied flip angles were $10-45^{\circ}$ (interval of $5^{\circ}$ ). Then the maximum SI resulted by each concentration of nanoparticles was measured. Linear relationship between SI and nanoparticle concentration was evaluated regarding square correlations of 0.95 and 0.99. Coil non-uniformity was considered to obtain accurate SI of each image.

Results: The maximum SI was obtained at the highest applied flip angle $\left(45^{\circ}\right)$. The linear relationship between SI and nanoparticle concentration was seen up to 112.21 and $98.83 \mu \mathrm{mol} \mathrm{Fe} / \mathrm{L}$ for the short $\left(10^{\circ}\right)$ and the long $\left(45^{\circ}\right)$ flip angles, respectively $\left(\mathrm{R}^{2}=0.95\right)$. These values were reduced up to 48.54 and $42.73 \mu \mathrm{mol} F$ / $\mathrm{L}$ for these flip angles with $\mathrm{R}^{2}$ of 0.99 .

Conclusions: The maximum SI will be increased at higher flip angles with non-linear relationship between SI and nanoparticle concentration. The result shows that an increase in the flip angle leads to a decrease in the range of the linearity. The optimum flip angle which is suitable for perfusion measurement was obtained at $10^{\circ}$ for our imaging parameters and sequence. The results of this study may be used in in vivo perfusion measurements.
\end{abstract}

Keywords:Iron oxide nanoparticles; Magnetic Resonance Imaging; Inversion Recovery

\section{Background}

Ultrasmall superparamagnetic iron oxide (USPIO) nanoparticles have been used as a blood pool (intravascular) contrast agent. They are useful for magnetic resonance angiography (MRA), assessing myocardial and renal perfusion, and myocardial injury (1-3). Intravascular contrast agents have higher relaxivity than the extracellular agents (4). Chambon et al. reported that T1 relaxivity of iron oxide nanoparticles is 5 to 6 times higher than the gadolinium chelates (5). The USPIO nanoparticles also have long blood half-life and provide adequate time for data acquisition in MR imaging. Therefore, intravascular signal intensity (SI) loss is little (6). SI of MR images depends on the magnetic field strength (7), size (8) and coating type (9) of the nanoparticles, concentration of the contrast agent such as gadolinium-diethylenetriamine penta-acetic acid (Gd-DTPA) $(10,11)$ or iron oxide nanoparticles (9), and imaging pulse sequences (e.g. spin echo, fast spin echo, and inversion recovery) and parameters (e.g. repetition time (TR), echo time (TE), inversion time (TI), and saturation time (TS) (12-18).

Previous studies using iron oxide nanoparticles showed that the flip angle exerts an important effect on the SI of the MR images and contrast of MRA (4). Different studies were performed to evaluate the flip angle effect on the MRA image quality (19) and to determine appropriate flip angle for MRA using iron oxide nanoparticles and different MRI pulse sequences $(20,21)$. They reported different flip angle values as the appropriate flip angle that leads to highest MR signal intensity for MRA. Anzai et al. reported little flip angle dependence on the image quality for abdominal MRA using USPIO nanoparticles (19). A study carried out by Mayo-Smith et al. showed that the maximum SI in blood samples occurred at flip angles of $30^{\circ}$ and $60^{\circ}$ before and after administration of AMI-227,

Copyright ( ) 2015, Tehran University of Medical Sciences and Iranian Society of Radiology. This is an open-access article distributed under the terms of the Creative Commons Attribution-NonCommercial 4.0 International License (http://creativecommons.org/licenses/by-nc/4.0/) which permits copy and redistribute the material just in noncommercial usages, provided the original work is properly cited. 
respectively (20). Tanimoto et al. found the optimal flip angle of $20^{\circ}$ for the inferior vena cava before and after USPIO injection (21).

Although evaluation of flip angle effect on SI and determination of appropriate flip angle has been investigated for MRA, in current MR perfusion studies, optimum flip angle has not been determined. For accurate perfusion measurement, it is important to find a flip angle that leads to the maximum linearity at the highest concentration to improve signal to noise ratio (SNR). The investigators still use different flip angles and pulse sequences for their perfusion measurement studies. However, it has not been cleared yet that which flip angle is optimum when we use the inversion recovery pulse sequence with an inversion time(TI) that suppresses the blood signal for in vitro situation.

\section{Objectives}

The aim of this study was to investigate the effect of flip angle on the linear relationship between SI and different concentrations of iron oxide nanoparticles in T1-weighted MR images using inversion recovery pulse sequence to find the optimum flip angle which is suitable for perfusion measurement. In addition, the maximum SI at different concentrations and different flip angles was also investigated using USPIO nanoparticles with carboxydextran coating.

\section{Materials and Methods}

\subsection{Theory}

The relationship between SI and imaging parameters in the inversion recovery sequence can be written as (12):

$$
S(t)=S_{0}\left(1-2 \exp \frac{-\mathrm{TI}}{T 1}+\exp \frac{-\mathrm{TR}}{T 1}\right) \exp \left(-\frac{\mathrm{TE}}{\mathrm{T} 2}\right)
$$

Where $S(t)$ is the SI after administration of the contrast agent, and So is the observed SI when there is no contrast agent. T1, TE, and T2 denote the tissue longitudinal relaxation time, the echo time and transverse relaxation time, respectively (15).

The relationship between SI and concentration of contrast agent is described by the following equation:

$$
S(t)=S_{0}\left(1-2 \exp \left(-\operatorname{TI}\left(\frac{C(t)}{K}+\frac{1}{T 1_{\mathrm{rr}}}\right)\right)+\exp \left(-\operatorname{TR}\left(\frac{C(t)}{K}+\frac{1}{T 1_{\mathrm{prc}_{\mathrm{re}}}}\right)\right)\right) \exp \left(-\frac{\mathrm{TE}}{T 2}\right)
$$

Where $\mathrm{T}_{\text {Pre }}$ is the longitudinal relaxation time before contrast application, $\mathrm{C}(\mathrm{t})$ is the concentration of the contrast agent at time $\mathrm{t}$, and $\mathrm{K}$ is a constant that depends on the contrast medium (15).

Equation 3 with respect to the effect of flip angle on SI can be written as (22):

$S(t)=S_{0}\left(1-2 \exp \left(-\operatorname{TI}\left(\frac{C(t)}{K}+\frac{1}{T 1_{\mathrm{Pr}}}\right)\right)+\exp \left(-\operatorname{TR}\left(\frac{\mathrm{C}(t)}{K}+\frac{1}{T 1_{\mathrm{Per}}}\right)\right)\right) \exp \left(-\frac{\mathrm{TE}}{T 2}\right) \times \operatorname{Sin}(\alpha)$
Where $\alpha$ is flip angle. It is necessary to mention that $\exp \left(-\frac{\mathrm{TE}}{T 2}\right)$ can be ignored from equations 1,2 , and 3 at lower concentrations of contrast agents. The T1-shortening effect is dominant at low concentrations. Therefore, the relationship between SI and concentration is linear. At high concentrations, both $\mathrm{T} 1$ and $\mathrm{T} 2$ can be affected as SI response becomes nonlinear with the concentration $(14,15)$.

\subsection{Contrast Agent}

Nanomag-D-spio nanoparticles (Micromod Partikeltechnologie $\mathrm{GmbH}$, Rostock, Germany) with a core size of $7 \mathrm{~nm}$, mean diameter of $20 \mathrm{~nm}$ and carboxydextran coating were used for this study. The concentration of iron in the ferro-fluid was measured as $1.29 \mathrm{mg} / \mathrm{mL}$ using atomic absorption spectrometer (analytik jena, novAA $^{\circledR} 400$, Germany). Then $1.8 \mathrm{~mL}$ of the ferro-fluid was diluted using $48.2 \mathrm{~mL}$ deionized water to prepare $50 \mathrm{~mL}$ of the highest concentration $(500 \mu \mathrm{mol} \mathrm{Fe} / \mathrm{L})$. Other concentrations were prepared with dilution of this sample with appropriate volumes of deionized water. Concentration of the nanoparticles varied between 0 and $500 \mu \mathrm{mol}$ $\mathrm{Fe} / \mathrm{L}(0-100 \mu \mathrm{mol} \mathrm{Fe} / \mathrm{L}$ with interval of $5 \mu \mathrm{mol} \mathrm{Fe} / \mathrm{L}$ and 100$500 \mu \mathrm{mol} \mathrm{Fe} / \mathrm{L}$ with interval of $100 \mu \mathrm{mol} \mathrm{Fe} / \mathrm{L})$.

\subsection{Phantom}

A Perspex phantom was designed with dimensions of 13 $\times 13 \times 13 \mathrm{~cm}$ to placement of glass via ls (internal diameter $=15 \mathrm{~mm}$ ) filled with various concentrations or constant concentrations of iron oxide nanoparticles (Figure 1). The phantom consisted of 25 vials with different and constant concentrations of nanoparticles that were carefully placed in the center of the standard clinical MRI head coil. Figure 1 shows a coronal image of the phantom with different concentrations.

Non-uniformity of the coil is a major challenge in MRI. To measure the accurate SI of an MR image, the response of the RF coil should be uniform. Therefore, 25 vials with constant concentration of iron oxide nanoparticles (50 $\mu \mathrm{mol} \mathrm{Fe} / \mathrm{L}$ ) were placed in exactly the same positions of the vials with different concentrations to measure the coil non-uniformity. The SI of one vial in the center of the coil was chosen as reference SI (e.g., the vial with concentration of $60 \mu \mathrm{mol} \mathrm{Fe} / \mathrm{L}$ ). The SI of all vials with the constant concentration should be equal to that SI. After normalization of the mean SI from the vials with constant concentration, the correction factor due to non-uniformity of the coil was calculated for different positions of the vials with different concentrations. To calculate the corrected SI for different concentrations, the SI of each vial was multiplied by its correction factor $(15,16)$. 
Gharehaghaji Net al.

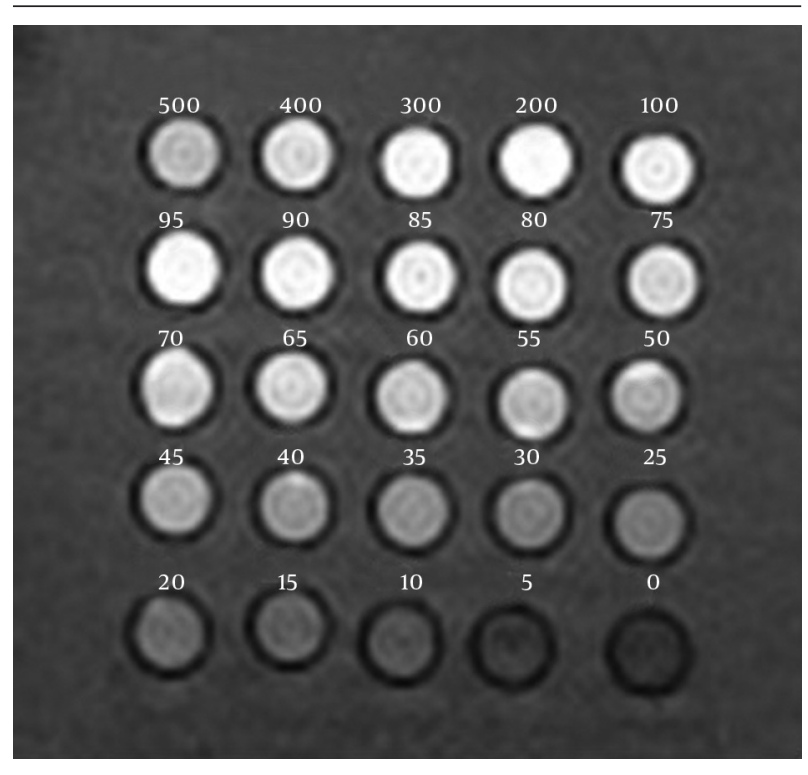

Figure 1. Coronal image of the phantom. The position of the different concentrations ( $\mu \mathrm{mol} \mathrm{Fe} / \mathrm{L}$ ) of nanoparticles is seen at the top of the vials

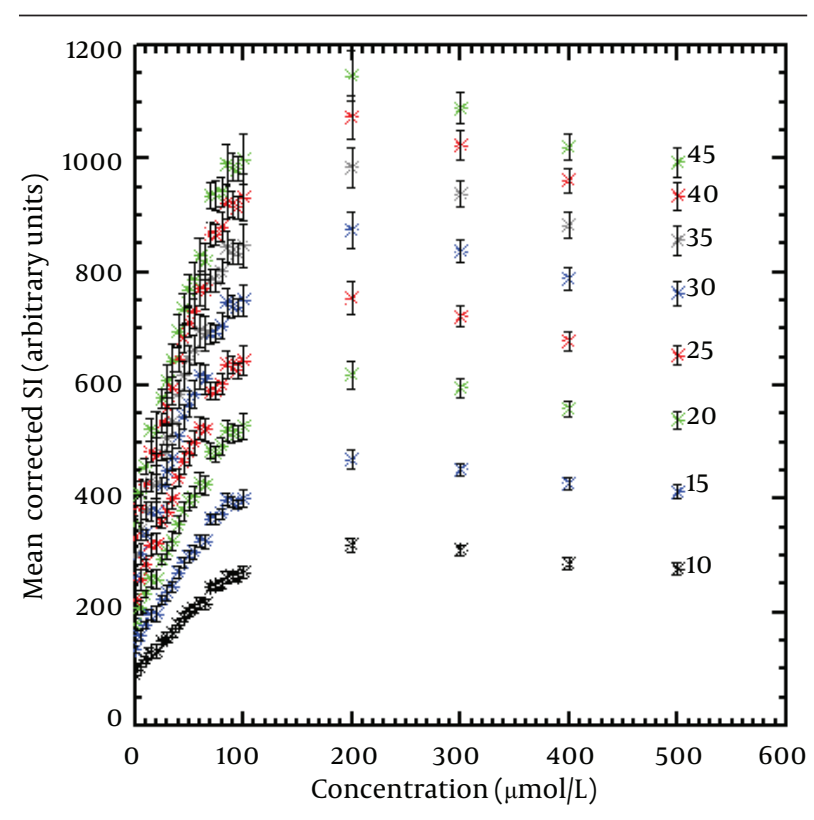

Figure 2. The mean corrected SI (for non-uniformity of the coil) from the 9 innermost pixels in each vial versus the concentrations of the nanoparticles from different flip angles $\left(10^{\circ}\right.$ to $\left.45^{\circ}\right)$. All maximum corrected SIs are seen at a concentration of $200 \mu \mathrm{mol} \mathrm{Fe} / \mathrm{L}$

\subsection{Image Acquisition}

MR imaging was performed using a 1.5 T MRI system (MAGNETOM Vision, Siemens, Avanto, Germany). T1weighted Turbo-FLASH IR sequence was used for this study. The imaging parameters were TR of $10 \mathrm{~ms}$, TE of $2.06 \mathrm{~ms}$, TI of $830 \mathrm{~ms}$, matrix size of $128 \times 128$, slice thickness of $10 \mathrm{~mm}$, and number of signal averaging (NSA) of 2. Flip angles were between 10 and $45^{\circ}$ (interval of $5^{\circ}$ ).
Table 1. The Maximum Corrected SI and Its Percentage for the Applied Flip Angles

\begin{tabular}{lcc}
\hline Flip Angle $\left(^{\circ}\right)$ & $\begin{array}{c}\text { Maximum } \\
\text { Corrected SI }\end{array}$ & $\begin{array}{c}\text { Maximum } \\
\text { Corrected SI (\%) }\end{array}$ \\
\hline $\mathbf{1 0}$ & 326.08 & 28 \\
$\mathbf{1 5}$ & 480.50 & 42 \\
\hline $\mathbf{2 0}$ & 633.67 & 55 \\
\hline $\mathbf{2 5}$ & 772.65 & 67 \\
\hline $\mathbf{3 0}$ & 893.48 & 78 \\
\hline $\mathbf{3 5}$ & 1006.19 & 88 \\
\hline $\mathbf{4 0}$ & 1097.26 & 96 \\
\hline $\mathbf{4 5}$ & 1146.59 & 100 \\
\hline
\end{tabular}

\subsection{Image Analysis}

The image data with DICOM format were transferred from the MR scanner to a personal computer. For data analysis, the image processing software interactive data language (IDL, Research Systems, Inc. http://www.rsinc. com) was used. The programs were written using IDL for the following purposes: 1) Measuring the mean SI of the 9 innermost pixels in the center of each vial for constant and different concentrations; 2) Calculating the correction factors related to coil non-uniformity; 3) Drawing the best-fit of SI versus concentration curves based on equation 3 ; 4) Finding the maximum linear correlation between the corrected SI and nanoparticles' concentrations at different flip angles with regard to square correlation coefficient $\left(\mathrm{R}^{2}\right)$ of 0.95 and 0.99 .

\section{Results}

The correction factors for non-uniformity of the coil were calculated for the vial positions with different concentrations. These factors varied from 0.99 to 1.18 . The SI of the vials with different concentrations was multiplied by these factors to obtain the corrected SI. Figure 2 shows concentration of the contrast agent versus the mean corrected SI for flip angles of $10-45^{\circ}$. The error bars show the standard deviation of SI from the 9 innermost pixels in each vial. All maximum corrected SIs for different flip angles were seen at a concentration of $200 \mu \mathrm{mol} \mathrm{Fe} / \mathrm{L}$. However, the highest corrected SI was obtained with the highest applied flip angle $\left(45^{\circ}\right)$. After this concentration, the corrected SI was gradually decreased for all flip angles.

The maximum corrected SIs for the applied flip angles are seen in Table 1 . The table shows that an increase in the flip angle led to an increase in the maximum corrected SI. Assuming the maximum corrected SI at the highest applied flip angle $\left(45^{\circ}\right)$ is $100 \%$, its value for other flip angles were calculated as Table 1.

The maximum linear relationship between corrected SI 
Gharehaghaji N et al.

\begin{tabular}{|c|c|c|}
\hline Flip Angle $\left({ }^{\circ}\right)$ & $\begin{array}{l}\text { Concentrations of the Nanoparticles that Give } R^{2}= \\
\qquad 0.95(\mu \mathrm{mol} \mathrm{Fe} / \mathrm{L})\end{array}$ & $\begin{array}{c}\text { Concentrations of the Nanoparticles that Give } \mathrm{R}^{2} \\
=0.99(\mu \mathrm{mol} \mathrm{Fe} / \mathrm{L})\end{array}$ \\
\hline 10 & 112.21 & 48.54 \\
\hline 15 & 109.50 & 47.45 \\
\hline 20 & 108.66 & 46.99 \\
\hline 25 & 106.64 & 46.12 \\
\hline 30 & 105.52 & 45.63 \\
\hline 35 & 103.663 & 44.83 \\
\hline 40 & 101.093 & 43.71 \\
\hline 45 & 98.83 & 42.73 \\
\hline
\end{tabular}

and the concentrations of the nanoparticles that gives $\mathrm{R}^{2}$ equal to 0.95 and 0.99 for the different flip angles is seen in Table 2. The linear relationship was seen up to 112.21 and $98.83 \mu \mathrm{mol} \mathrm{Fe} / \mathrm{L}$ for the short $\left(10^{\circ}\right)$ and the long $\left(45^{\circ}\right)$ flip angles, respectively with $\mathrm{R}^{2}$ of 0.95 . These values were reduced up to 48.54 and $42.73 \mu \mathrm{mol} \mathrm{Fe} / \mathrm{L}$ for these flip angles with $\mathrm{R}^{2}$ of 0.99 . Decreasing the concentration of the nanoparticles that led to the maximum linear corrected SI was seen by increasing the flip angle values.

\section{Discussion}

The perfusion parameters such as cerebral blood flow, cerebral blood volume, and mean transit time can be obtained from concentration-time curve after contrast agent administration. MRI measures SI and is unable to measure the concentration of the contrast agent directly. Therefore, it is only possible to achieve SI-time curve with MRI. If the relationship between SI and concentration is to be linear over a range of the concentrations, then relative changes in SI can be used instead of concentration. $\mathrm{R}^{2}$ gives the strength of the linear relationship between SI and concentration. When $\mathrm{R}^{2}$ is 0.99 , it indicates that $99 \%$ of the variation in SI is explained by the variation of concentration (23).

According to equation 3 and Figure 2, The T1-shortening effect was dominant at low concentrations. Therefore, the relationship between SI and concentration was linear. At high concentrations, both $\mathrm{T} 1$ and T2 (with dominance of T2) had effect on the SI response and therefore, it became nonlinear with the concentration. The effect of T1-shortening led to increase in SI, whereas the T2-shortening effect became dominant at high concentrations (more than $200 \mu \mathrm{mol} \mathrm{Fe} / \mathrm{L}$ ) and led to decrease in SI (Figure 2). The linear parts of all curves were found at the beginning of the curves where the T1-shortening effect was dominant. The linearity of the correlation between mean corrected SI and nanoparticles' concentration decreased as flip angle increased. However, as seen in Table 2, the differences among linearity values for different flip angles were low for both $\mathrm{R}^{2}$ of 0.95 and 0.99 . Since $\mathrm{R}^{2}$ indicates the strength of the linear correlation between SI and con- centration, the range of the linearity values was lower for $\mathrm{R}^{2}$ of 0.99 than $\mathrm{R}^{2}$ of 0.95 .

According to our knowledge, there was no research about evaluating flip angle effect on the linear relationship between SI and different concentrations of iron oxide nanoparticles to compare with the results of this study. As seen in Figure 2 Table 1, an increase in flip angle led to an increase in maximum corrected SI based on equation 3. Therefore, among different applied flip angles, the highest corrected SI was seen at flip angle of $45^{\circ}$. At lower flip angles $\left(10-20^{\circ}\right)$, the increase in SI was small due to the increase of the T2-shortening effect. On the other hand, if we assume that the maximum corrected SI resulted by the highest applied flip angle $\left(45^{\circ}\right)$ is $100 \%$, SI for other flip angles will be calculated as shown in Table 1. This finding indicates that the effect of flip angle increase on the maximum corrected SI is relatively higher for low flip angles (14\% maximum corrected SI difference between flip angles of $10^{\circ}$ and $15^{\circ}$ against $4 \%$ difference between flip angles of $40^{\circ}$ and $45^{\circ}$ ). This point is important in clinical studies where decreasing of scan time is needed using lower TRs and flip angles. Although the maximum corrected SIs for all applied flip angles were seen at a concentration of $200 \mu \mathrm{mol} \mathrm{Fe} / \mathrm{L}$, there was a considerable difference in the maximum corrected SI when the flip angles varied from $10^{\circ}$ to $45^{\circ}$ ( $72 \%$ ).

Investigation of the flip angle effect on SI of the blood samples in a T1-weighted gradient-echo sequence before and after administration of USPIO nanoparticles (AMI227) was carried out by Mayo-Smith et al. (20). They reported that the maximum SI occurred at flip angles of $30^{\circ}$ and $60^{\circ}$ for before and after administration of AMI227, respectively. Although the nanoparticle size in the study conducted by Mayo-Smith et al. and our study was approximately similar, the maximum SI in our study was found at flip angle of $45^{\circ}$ for both absence of the nanoparticles (concentration of 0 ) and presence of different concentrations of the particles. The different results can be related to using different imaging parameters and sequences.

Tanimoto et al. (21) evaluated the effect of iron oxide nanoparticles in three-dimensional phase-contrast MR 
angiography in the rat using flip angles of $20^{\circ}, 30^{\circ}$, and $40^{\circ}$. They reported the optimal flip angle of $20^{\circ}$ for the inferior vena cava before and after USPIO injection. On the other hand, the results of another study (19) showed a little flip angle dependence on the image quality for abdominal MRA using USPIO nanoparticles. Since each material has its own special $\mathrm{T} 1$ relaxation time, and the flip angle that gives maximum SI is a function of T1 and TR; therefore, it is not possible to compare other investigators' results with ours. In addition, our research was an in vitro study and also imaging parameters and sequences in this study were different than those of other investigators. Therefore, our results cannot be compared with their findings.

This study shows that flip angle is one of the important parameters for measuring SI. It is well known that increase of the flip angle leads to increase in maximum SI, but for perfusion measurement it is important to find a flip angle that leads to the maximum linearity at the highest concentration to improve SNR. Since the volume of injected contrast agent in T1-weighted is about 1/10th of the normal suggested dose in $\mathrm{T} 2$-weighted perfusion imaging (23), the use of small volumes of nanoparticles results in reduced SNR. This ratio should be improved using more concentration of contrast agent.

This study also shows that the flip angle has an effect on the maximum linear relationship between SI and concentration. An increase in flip angle leads to a decrease in the maximum linearity (Table 2). Therefore, a flip angle of $10^{\circ}$ is the optimum flip angle for perfusion measurement with our imaging parameters and sequence. Since $\mathrm{TI}=830 \mathrm{~ms}$ in Turbo-FLASH IR sequence is used in clinical studies because of the null point signal from blood, the results of this study may be used for the perfusion measurements $(23,24)$.

In conclusion, the effect of flip angle on the relationship between SI and different concentrations of iron oxide nanoparticles was evaluated using T1-weighted TurboFLASH inversion recovery sequence to find the optimum flip angle that is suitable for perfusion measurement. This study shows that a difference in flip angle has an effect on both maximum SI, and the linear relationship between SI and nanoparticle concentration. The maximum SI will increase at higher flip angles with non-linear relationship between SI and the concentrations of the nanoparticles. The result indicates that an increase in the flip angle leads to a decrease in the range of the linearity. The optimum flip angle that is suitable for perfusion measurement was obtained at $10^{\circ}$ for our imaging parameters and sequence. Since this study was performed using a routine 1.5 T MRI system and a TI which suppresses blood signal, the results of this study may be used in in vivo study for perfusion measurements.

\section{Acknowledgements}

Data acquisition was performed in Ali Nasab Eram Hospital, which is gratefully acknowledged.

\section{Authors' Contributions}

All authors participated equally in this study.

\section{Funding/Support}

This work was financially supported by Tabriz University of Medical Sciences.

\section{References}

1. Reimer P, Bremer C, Allkemper T, Engelhardt M, Mahler M, Ebert W, et al. Myocardial perfusion and MR angiography of chest with SH U 555 C: results of placebo-controlled clinical phase i study. Radiology. 2004;231(2):474-81.

2. Bachmann R, Conrad R, Kreft B, Luzar O, Block W, Flacke S, et al. Evaluation of a new ultrasmall superparamagnetic iron oxide contrast agent Clariscan, (NC100150) for MRI of renal perfusion: experimental study in an animal model. J Magn Reson Imaging. 2002;16(2):190-5.

3. Krombach GA, Wendland MF, Higgins CB, Saeed M. MR imaging of spatial extent of microvascular injury in reperfused ischemically injured rat myocardium: value of blood pool ultrasmall superparamagnetic particles of iron oxide. Radiology. 2002;225(2):479-86.

4. Saeed M, Wendland MF, Higgins CB. Blood pool MR contrast agents for cardiovascular imaging. J Magn Reson Imaging. 2000;12(6):890-8.

5. Chambon C, Clement O, Le Blanche A, Schouman-Claeys E, Frija G. Superparamagnetic iron oxides as positive MR contrast agents: in vitro and in vivo evidence. Magn Reson Imaging. 1993;11(4):509-19.

6. Li W, Tutton S, Vu AT, Pierchala L, Li BS, Lewis JM, et al. First-pass contrast-enhanced magnetic resonance angiography in humans using ferumoxytol, a novel ultrasmall superparamagnetic iron oxide (USPIO)-based blood pool agent. J Magn Reson Imaging. 2005;21(1):46-52.

7. Nazarpoor M, Mayabi Z, Shfaie A, Pesianian E, Aghaverdizadeh D. [Maximum relationship between signal intensity and concentration of contrast agent in $0.3 \mathrm{~T}$ and $1.5 \mathrm{~T}$ using T1-weighted spin echo sequence]. Med J Tabriz Uni Med Sci \& Heal Serv. 2011;32:72-6.

8. Allkemper T, Bremer C, Matuszewski L, Ebert W, Reimer P. Contrast-enhanced blood-pool MR angiography with optimized iron oxides: effect of size and dose on vascular contrast enhancement in rabbits. Radiology. 2002;223(2):432-8.

9. Oghabian MA, Gharehaghaji N, Masoudi A, Shanehsazzadeh S, Ahmadi R, Faridi MR, et al. Effect of coating materials on lymph nodes detection using magnetite nanoparticles. Adv Sci Eng and Med.2013;5(1):37-45.

10. Nazarpoor M. Inflow effect of signal intensity for the centre out phase-encoding and linear phase-encoding acquisitions on inversion recovery T1-weighted TurboFLASH images. J Cardiovasc Thorac Res. 2009;1:29-37.

11. Nazarpoor M, Poureisa M, Daghighi MH. [Investigations of optimal dose of contrast agent concentration from routine dose using spin echo and inversion recovery T1-weighted sequences in MRI]. Med J Tabriz Uni Med Sci \& Heal Serv. 2013;34:74-8.

12. Nazarpoor M, Poureisa M, Daghighi MH. Comparison of maximum signal intensity of contrast agent on t1-weighted images using spin echo, fast spin echo and inversion recovery sequences. Iran J Radiol. 2012;10(1):27-32.

13. Gharehaghaji N, Oghabian MA, Sarkar S, Amirmohseni S, Ghanaati $\mathrm{H}$. Optimization of pulse sequences in magnetic resonance lymphography of axillary lymph nodes using magnetic nanoparticles. J Nanosci Nanotechnol. 2009;9(7):4448-52.

14. Nazarpoor M. The effect of repetition time on the maximum linear relationship between contrast agent concentration and signal intensity on T1-weighted image using inversion recovery (IR) sequence. Iran J Radiol. 2009;6(4):247-52.

15. Nazarpoor M. Effects of inversion and saturation times on relationships between contrast agent concentrations and signal intensities of T1-weighted magnetic resonance images. Radiol Phys Technol. 2010;3(2):120-6. 


\section{Gharehaghaji N et al.}

16. Saharkhiz H, Gharehaghaji N, Nazarpoor M, Mesbahi A, Pourissa M. The Effect of Inversion Time on the Relationship Between Iron Oxide Nanoparticles Concentration and Signal Intensity in T1Weighted MR Images. Iran J Radiol. 2014;11(2).

17. Nazarpoor M, Poureisa M, Daghighi MH. Effect of echo time on the maximum relationship between contrast agent concentration and signal intensity using FLAIR sequence. Iran J Med Phys. 2013:1-2.

18. Oghabian MA, Guiti M, Haddad P, Gharehaghaji N, Saber R, Alam $\mathrm{NR}$, et al. Detection sensitivity of MRI using ultra-small super paramagnetic iron oxide nano-particles (USPIO) in biological tissues. Conf Proc IEEE Eng Med Biol Soc. 2006;1:5625-6.

19. Anzai Y, Prince MR, Chenevert TL, Maki JH, Londy F, London M, et al. MR angiography with an ultrasmall superparamagnetic iron oxide blood pool agent. J Magn Reson Imaging. 1997;7(1):209-14.

20. Mayo-Smith WW, Saini S, Slater G, Kaufman JA, Sharma P, Hahn PF MR contrast material for vascular enhancement: value of super- paramagnetic iron oxide. AJR Am J Roentgenol. 1996;166(1):73-7.

21. Tanimoto A, Yuasa Y, Hiramatsu K. Enhancement of phase - contrast MR angiography with superparamagnetic iron oxide. $J$ Magn Reson Imaging. 1998;8(2):446-50.

22. Thomas DL, De Vita E, Deichmann R, Turner R, Ordidge RJ. 3D MDEFT imaging of the human brain at $4.7 \mathrm{~T}$ with reduced sensitivity to radiofrequency inhomogeneity. Magn Reson Med. 2005;53(6):1452-8.

23. Nazarpoor M, Morgan PS. Inflow effect correction in perfusion measurement of normal subjects with T1-weighted images using inversion recovery sequences. Iran J Radiol. 2010;7(3):145-51.

24. Nazarpoor M. Organ Blood Flow Measurement with T1 and T2* Weighted MRI Techniques . Saarbrücken: LAP LAMBERT Academic Publishing GmbH \& Co; 2012. Organ Blood Flow Measurement with $\mathrm{T} 1$ and $\mathrm{T} 2 *$-Weighted MRI Techniques. 